\title{
Déclaration anonyme informatisée d'incidents critiques: une contribution à la sécurité des patients
}

\author{
M. Kaufmann ${ }^{a}$, S. Staender ${ }^{b}$, G. von Below ${ }^{c}$, H. H. Brunner ${ }^{c}$, L. Portenier ${ }^{\text {, D. D. Scheidegger }}{ }^{a}$
}

Deutsch erschienen

in Nr. 47/ 2002.

a Département d'anesthésie de l'Hôpital cantonal de Bâle

b Département d'anesthésie, Hôpital de Männedorf

c Fédération des médecins suisses (FMH)

d Association suisse des infirmières et infirmiers (ASI)

Correspondance

Dr M. Kaufmann, p.-d.

Département d'anesthésie

Cliniques universitaires (KBS)

CH-4031 Bâle

\section{Résumé}

Dans le passé, on n'a tenu qu'insuffisamment compte de la sécurité des patients en tant que part essentielle du processus thérapeutique. Un élément crucial de l'augmentation de la qualité est la création d'une culture dans ce domaine qui ait pour effet d'identifier les erreurs et de mettre à jour les causes possibles et les mesures à prendre pour les éviter à l'avenir. L'expérience rassemblée dans divers secteurs a montré que la collecte systématique d'incidents critiques (Critical Incident Reporting) est un élément important de sécurité et d'apprentissage. Les systèmes d'annonces volontaires anonymes présentent, à l'encontre des systèmes d'annonces obligatoires, un potentiel de mise à jour plus étendue des défaillances et des fautes du système avant l'apparition de complications plus graves.

L'article ci-après présente un système bénéficiant d'un large soutien (médecins, infirmières et infirmiers, FMH, pharmaciens) sur la base du système de déclaration d'incidents critiques (CIRS) déjà en place depuis des années dans le domaine de l'anesthésie. Ce système CIRSmedical (installé sur ordinateur, intranet ou internet) donne indirectement un nombre minimal de données tout en ayant un potentiel d'adaptation spécifique au groupe ou à la discipline. Le système est gratuitement mis à la disposition des groupes intéressés (www.CIRSmedical.org). Il a pour objectif de diminuer les réticences qui pourraient surgir à l'introduction de tels systèmes. Ces derniers peuvent être mis en place dans les hôpitaux de manière locale, décentralisée ou propagée à des groupes fermés d'utilisateurs.

\section{Introduction}

La sécurité des patients est un objectif important de nos systèmes modernes de santé publique. On a dû constater que nombre de patients ont subi involontairement un dommage dans le cadre d'une assistance médicale de routine [1, 2]. Des données émanant de plusieurs pays indiquent que si la plu- part de ces incidents demeurent sans graves conséquences, près de $1 \%$ des cas se retrouvent avec des dommages importants, voire mortels. Il est important de relever que près de la moitié de ces événements porteurs de conséquences défavorables se fondent sur des incidents critiques (critical incidents) en principe évitables [3]. Si l'on songe qu'un grand hôpital traite plus de 100000 patients par année, sur le plan ambulatoire et hospitalier, on voit d'emblée l'importance factuelle et réelle de ce problème et quelles en sont les conséquences sur les coûts pour la santé publique et la société (prolongation du séjour hospitalier, dommages permanents, réduction de la capacité de travail, responsabilité civile ...).

Il convient de relever que ces quelques incidents critiques sont dus trop souvent à de fausses manœuvres ou à des défaillances (erreurs humaines). Leur analyse, tout comme l'expérience rassemblée dans d'autres domaines (aviation, industrie nucléaire et pétrolière ou autres infrastructures complexes), a cependant clairement montré qu'ils sont le plus souvent d'origine multifactorielle: surcharge de travail, problèmes de communication, de formation, de surveillance, ressources insuffisantes, problèmes d'équipe, environnement inadéquat et, notamment, problèmes dus aux patients eux-mêmes $[4,5]$. Dans le domaine de la santé publique suisse, les stratégies préventives en la matière en sont encore à leurs balbutiements, contrairement aux stratégies claires déjà en usage à l'étranger (tab. 1). Rappelons à cet égard que, dans le passé, ce sont surtout les tenants des disciplines médicales spécialisées (p. ex. l'anesthésiologie) qui se sont penchés très tôt sur ce sujet, reprenant et adaptant des méthodes de sécurité déjà en usage ailleurs (p. ex. en aéronautique) [6, 7].

\section{Système de déclaration d'incidents critiques: apprendre sur la base des fautes commises}

D’une manière générale, les incidents critiques (IC) sont des événements ou des circonstances 
Tableau 1

Eléments-clé des stratégies de prévention à l'étranger.

\begin{tabular}{|l|l|}
\hline GB National Health Service (NHS) & US Institute of Medicine \\
\hline $\begin{array}{l}\text { - Team based training with recognition } \\
\text { that AE lessons may be relevant for others }\end{array}$ & $\begin{array}{l}\text { - Enhance knowledge about safety } \\
- \text { Mandatory reporting of adverse events } \\
\text { - Potential for repetition of similar adverse } \\
\text { events is recognised }\end{array}$ \\
$\begin{array}{l}\text { - Blame free reporting } \\
\text { - Coordinated database with conscientious } \\
\text { use of data }\end{array}$ & $\begin{array}{l}\text { - Implementation of safe practices } \\
\text { at the provider level }\end{array}$ \\
- Feedback to provider & \\
- Active learning & \\
- Systems approach to prevention & \\
- Risk management awareness & \\
\hline
\end{tabular}

ayant (ou pouvant avoir) une influence négative (ou positive) sur les objectifs d'un système. Dans le domaine de la santé publique, les définitions sont nombreuses [11]. Pour notre part, nous entendons par là des événements qui sans intervention humaine auraient pu aboutir (ou, en dépit de celle-ci), ont abouti à un événement indésirable, à savoir à un dommage physique ou psychique du patient. Cela revient à dire qu'il s'agit d'incidents dont l'origine est à attribuer essentiellement à une marge de sécurité diminuée et non pas à un accident, une complication ou une circonstance défavorable.

Les graves incidents et complications sont relativement rares. Il ne serait guère utile de vouloir améliorer la sécurité du système par des études pointilleuses de tels cas rarissimes alors qu'il peut se produire chaque jour, dans le travail en santé publique également, des micro-événements porteurs de conséquences négatives, à savoir des incidents critiques ou IC. Dans certaines circonstances, ces IC peuvent déborder sur une

Figure 1

Modèle genre «fromage suisse» du système de déclaration d'incidents critiques: les systèmes modernes sont fondés pour la plupart sur des processus de barrières de sécurité (systèmes d'alarme, prescriptions, contrôles, normes, mesures physiques, etc.). Ces barrières ne sont pas infaillibles. II est rare que ces lacunes produisent un enchaînement de circonstances aboutissant à un incident critique. Les incidents critiques peuvent permettre d'identifier les lacunes des diverses barrières de sécurité.

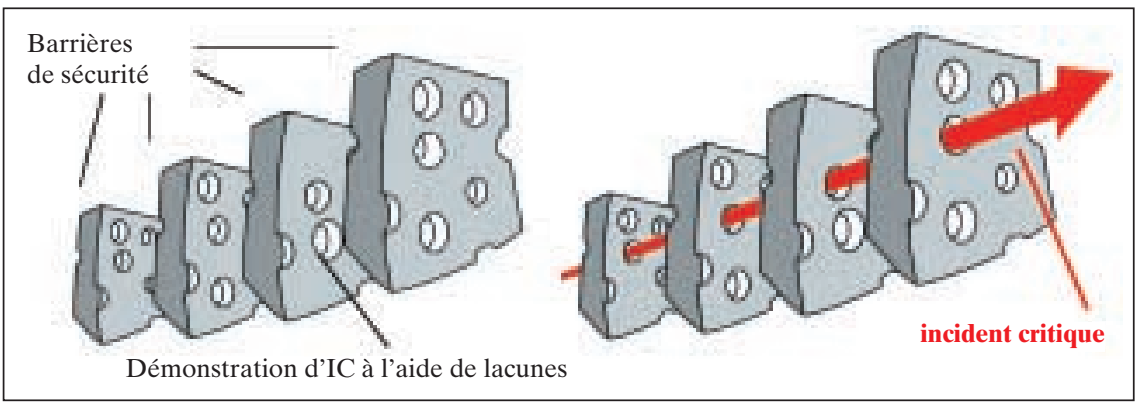

complication. Vu la fréquence élevée d'IC dans le quotidien clinique, leur analyse forme une base nettement plus solide pour améliorer les efforts mis à la sécurité du système. Ces incidents mettent à jour les grandes et petites lacunes en matière de sécurité. Les combler accroît la sécurité dans son ensemble. La technique des IC se distingue par le fait que la collecte d'informations sur les incidents permet de corriger ce qu'il y a à corriger («combler les lacunes de sécurité») et d'éviter ainsi de futures erreurs [8, 9] (Adverse Events). (Fig. 1).

Les déclarations établies sur de tels incidents sont, en Suisse également, aujourd'hui un élément central du management des risques, comme ce fut à nouveau relevé lors du congrès suisse intitulé «Vers un système de santé sécurisé», avec la proposition de mise sur pied d'un programme national pour améliorer la sécurité du patient en Suisse (Lucerne, 4/2001). [10]

\section{Avantages des déclarations d'IC $[7,9,11]$}

- Les incidents critiques sont en général rapidement répertoriés par les personnes directement concernées, sur les lieux de l'événement.

- Le recensement de tels événements peut se faire de manière structurée. Les conséquences qui en sont tirées peuvent notamment être utilisées afin de renforcer les éléments positifs du système.

- Technique avantageuse au sein de l'entreprise avec haute teneur informative.

- Technique qui met tout particulièrement en évidence les éléments perturbateurs du système.

- Technique qui sied tout particulièrement aux événements rares et atypiques.

- Technique utile aussi en tant que collectrice de déclarations de risques d'incidents, même si elle n'est pas l'objectif premier d'une système fondé sur les IC.

- Cette technique permet l'accès à des stratégies de gestion et de prévention des IC.

\section{Inconvénients des déclarations d'IC}

- Un problème réside dans le fait que seuls les événements réalisés de manière consciente et inscrits dans la mémoire peuvent être admis dans un tel système.

- Une autre condition est l'exactitude et la vérité des déclarations.

- La technique des IC met en évidence les événements plutôt rares, alors que les événements très fréquents, «banals», sont davantage occultés. En principe, la déclaration d'une partie seulement des événements se 
Tableau 2

Regard sur I'histoire de la déclaration d'IC et de son application électronique.

\begin{tabular}{|c|c|}
\hline 1965 & Hubbard: Clinical competence of residents (N Engl J Med) \\
\hline 1987 & Runciman: Australian Incident Monitoring study in Anaesthesia \\
\hline 1995 & $\begin{array}{l}\text { INTERNET + Incident Reporting in Anesthesia } \\
\rightarrow \text { CIRS }^{\odot} \text { worldwide (DA, Uni Basel: www.anaesthesie.ch/cirs/) }\end{array}$ \\
\hline 1998 & $\begin{array}{l}\text { CIRS }^{\odot}+\text { Société suisse d'anesthésiologie et de réanimation (SSAR) } \\
\rightarrow \text { CIRS-CH (SSAR: www.sgar-ssar.ch/qualitaet/) }\end{array}$ \\
\hline 1995 & $\mathrm{CIRS}^{\circ}+\mathrm{FMH} / \mathrm{ASI} \rightarrow \mathrm{CIRSmedical}^{\odot}$ (POPS-UHBS, FMH, ASI: www.CIRSmedical.ch) \\
\hline
\end{tabular}

produisant dans ce type d'environnement est un problème, notamment lorsque cette technique et le mode de pensée qui s'y rattachent n'ont été qu'insuffisamment intégrés (phénomène de l'iceberg).

\section{Application de la méthode}

Afin d'encourager un mode de déclaration unifié des incidents critiques dans le domaine de la santé - par delà les disciplines médicales - le groupe de sécurité périopératoire des patients des cliniques universitaires de Bâle (UHBS), sur la base de sa longue expérience (tab. 2) de travail commun avec la FMH, la Société professionnelle

Figure 2

Structure de la formule de déclaration électronique. Dans la partie «événement», on trouve les éléments de catégorisation automatiquement structurés selon les groupes professionnels et les domaines de responsabilité (médecine générale, chirurgie, gynécologie, médecine interne, anesthésie, etc.)

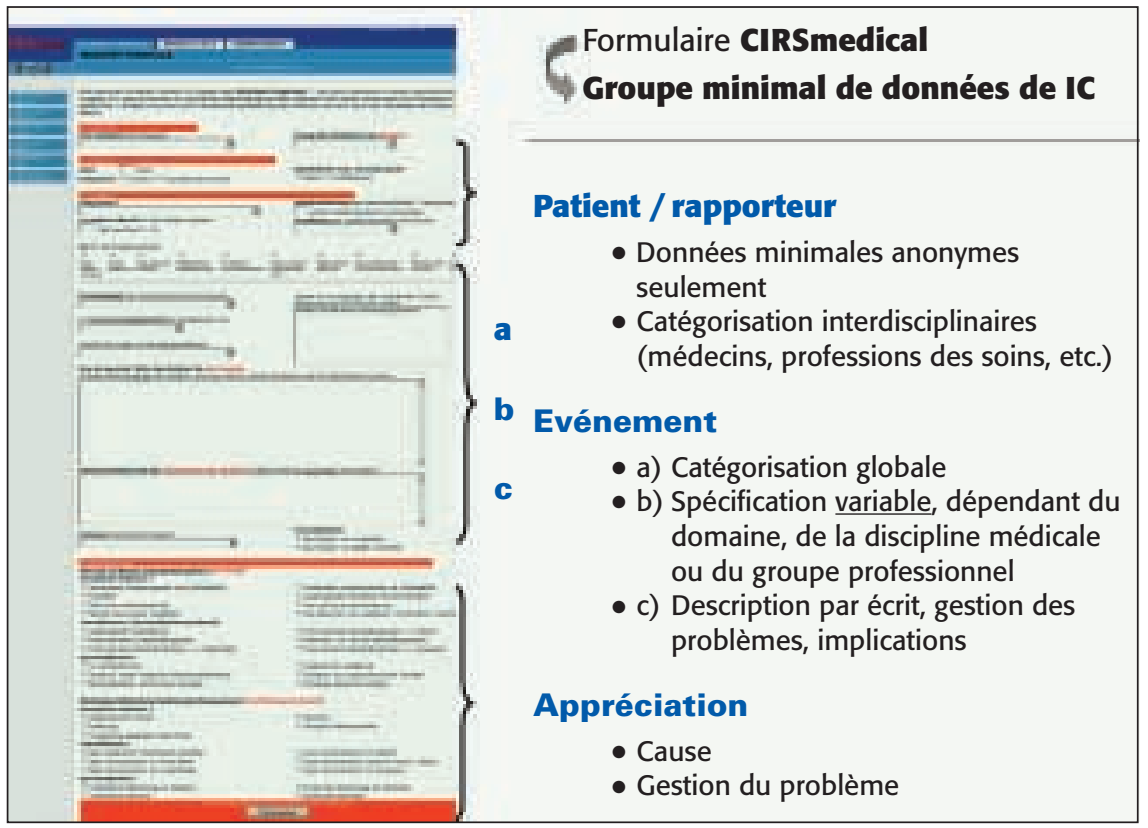

des pharmaciens d'administration et des hôpitaux (GSASA), l'Université de Bâle et l'Association suisse des infirmières et infirmiers (ASI), a défini les données minimales d'un système générique anonyme de déclaration des IC, accessible sur l'internet à l'adresse suivante: www.cirsmedical.ch. Ce système en quatre langues (f/d/i/a) se distingue par le fait qu'il réduit à un minimum les données du rapporteur des faits au quotidien tout en conservant une grande possibilité d'adaptation aux spécificités locales, ainsi qu'à celles des diverses professions et disciplines. (Fig. 2):

- Rapporteur: un rapporteur est adjoint au spécialiste du groupe professionnel concerné pour rendre compte de l'événement.

- Les données concernant le patient, pour autant qu'elles s'imposent, se réduisent à son âge et au mode de prise en charge (prévu ou en urgence).

- L'incident est rapporté de manière plus détaillée. Hormis la nature de l'incident (heure à laquelle il s'est produit, lieu de l'incident, charge de travail et groupe professionnel de son auteur, formation, domaine de responsabilité [discipline]), les éléments supplémentaires suivants sont pris en compte: Un médecin auteur d'un IC dans le domaine de la gynécologie/obstétrique, par exemple, aura le choix entre des données spécifiques issues des domaines suivants: gynécologie, ambulatoire, opératoire/anesthésie, endocrinologie, division pré-partum, salle d'accouchement, division post-partum, etc. Un soignant dans le domaine de l'anesthésie aura à disposition les éléments spécifiques à la médecine d'urgence préclinique, à l'anesthésie périopératoire, au traitement de la douleur, à la médecine intensive, etc. Il en va de même des domaines de responsabilité suivants: médecine générale, chirurgie, médecine interne, pédiatrie, psychiatrie, domaine diagnostique, pharmacologie, administration, entreprise, etc. Par ailleurs, l'IC est décrit de manière non structurée, à la manière de la description d'un cas, sans détails qui pourraient aboutir à identifier le patient ou le rapporteur. Il s'agit également de décrire le mode de gestion et les conséquences de la situation (à partir du moment de l'incident), de savoir à quel résultat on doit s'attendre et si l'incident était évitable.

- Dans l'évaluation de l'IC, on distingue entre le recensement structuré de l'origine de l'IC (quel événement y a-t-il conduit?) et la stratégie visant à y faire face (points les plus importants et les plus utiles dans la gestion de l'événement). 
La description de l'événement, sa gestion et les aspects relatifs aux résultats peuvent être discutés par tous les utilisateurs habilités à se servir du système, de manière anonyme et par voie électronique. Sous «évaluation», on peut directement visualiser les autres éléments du groupe de données minimal en tant que statistique globale. Il va de soi que l'utilisateur d'un tel système CIRSmedical de déclaration anonyme informatisée, local ou régional, a un accès illimité à cette banque de données afin de pouvoir procéder à des évaluations individuelles.

Pour les cercles fermés d'utilisateurs, il existe une application Windows avec un ensemble de fonctions librement accessible. Hormis ce simple système de rapport par voie électronique, ce mode interactif d'interprétation locale du rapport d'incidents contient également une analyse et des fonctions supplémentaires fondées sur les définitions spécifiques et actualisées que l'on trouve sur l'internet. Cette application autonome avec auto-installation peut être téléchargée à la seule condition d'envoyer régulièrement les incidents de manière anonymisée au centre collecteur pour y être analysés (CIRSmedical@ unibas.ch). Pour les institutions et les hôpitaux de plus grande importance disposant d'un grand trafic de données et pour les différents types de clients (PC, MAC, UNIX, LINUX, ...), l'application internet sur un serveur est également disponible.

Le système existant aussi bien en version place de travail (environnement Windows) qu'en version internet (tous les systèmes de gestion de la clientèle), on peut songer aux possibilités suivantes:

I. Utilisation interne au département par une unité centrale de traitement.

II. Utilisation sur l'intranet d'un hôpital ou d'un complexe hospitalier.

III. Utilisation strictement protégée par des groupes fermés d'utilisateurs au sein du système Health Info Net (HIN) de la FMH.

IV. Utilisation ouverte sur l'internet (www. CIRSmedical.org) par le biais de connexions SSL au serveur CIRSmedical ou d'un accès protégé par mot de passe à un cercle fermé d'utilisateurs.

La toile mondiale constitue le moyen idéal pour la déclaration électronique d'incidents critiques pour les raisons suivantes:

- accès à l'internet plus simple et plus étendu;

- anonymat systématisé du rédacteur de l'IC indépendant de la mise en œuvre concrète (cf. variante I à IV plus haut);
- structure uniformisée des rapports accessible par interface internet;

- possibilité de retour d'information directe et anonyme;

- large visualisation en ligne d'incidents existants: apprendre des autres (effet préventif);

- possibilité de discussion anonyme d'événements;

- centralisation des événements rares.

Plusieurs aspects (origine des données, protection relative vis-à-vis de l'extérieur) parlent en faveur des deux premières formes d'utilisation. La troisième variante concerne plus volontiers la large diffusion, les aspects juridiques et la couverture étendue à des disciplines médicales particulières (avec déclaration obligatoire selon accord), d'autant plus que le système permet, audelà des données minimales, d'ajouter à d'autres données spécifiques à l'hôpital ou à une discipline (version personnalisée).

Le logiciel mis à disposition offre d'autres possibilités:

- accès uniquement avec mot de passe;

- groupes séparés d'utilisateurs (mots de passe personnels);

- diffusion des incidents par les intermédiaires ou superviseurs locaux par présentation visuelle des données;

- possibilité de masquer les descriptions de cas «inadéquates»;

- possibilité de champs de saisie supplémentaires aux champs de données minimaux;

- mise à jour par téléchargement informatisé (dossier XML).

L'aspect juridique de CIRSmedical, ou la possibilité de recourir aux données de CIRSmedical dans les actions en justice n'est pas encore éclaircie. Actuellement, des représentants de la Faculté de droit de Bâle examine la question de savoir comment obtenir une interdiction d'utilisation des données de CIRSmedical lors de litiges devant les tribunaux. En principe, il convient néanmoins de relever que le mode de faire et la teneur des données rassemblées rendent pratiquement impossibles leur utilisation dans un cas concret, le rapport entre les données anonymisées et le patient ou le médecin étant de toute façon très difficile à faire, sauf lors d'une description très spécifique d'un cas, dans le cadre d'un cercle d'utilisateurs restreint (p. ex. système hospitalier local). 


\section{Evaluation}

La déclaration d'incidents critiques peut apporter un soutien efficace à l'apprentissage par les fautes, en aidant à mettre en évidence les lacunes potentiellement critiques. Les modifications de système se fondant sur les connaissances issues d'un système d'incidents critiques permettent d'augmenter la sécurité de manière active $[12,13]$. Le recours à un tel système conduit implicitement à un changement de mentalité par rapport aux erreurs dans l'environnement où il est appliqué. Une description unifiée, sur la base d'un ensemble des mêmes données minimales permet en principe une analyse des IC par-delà les disciplines et les groupes professionnels. Cela dit, la tendance relativement importante de minimisation des déclarations, due à la routine quotidienne du domaine clinique, fait que les informations ainsi recueillies devront être interprétées à l'avenir avec la plus grande prudence. L'utilité première du recours à un tel système d'IC réside certainement dans le fait de pouvoir influer localement sur le genre de fautes commises dans le cadre des divers systèmes de direction.

\section{Références}

1 Brennan TA, Leape LL, Laird NM, et al. Incidence of adverse events and negligence in hospitalized patients. N Engl J Med 1991;324:370-6.

2 Leape LL, Brennan TA, Troyen A, et al. The nature of adverse events in hospitalized patients: Results of the Harvard Medical Practice Study II. N Engl J Med 1991;324:377-84.

3 Wilson RM, Runciman WB, Gibberd RW, et al. The Quality in Australian Health Care Study. Med J Aust 1995;163:458-71.

4 Leape LL. Error in medicine. JAMA 1994;272:1851.

5 Reason JT. Human error: models and management. Br Med J 2000;320:768-70.

6 Staender S, Davies J, Helmreich B, Sexton B, Kaufmann M. The anaesthesia critical incident reporting system: an experience based database. Int J Med Inf 1997;47:87-90.

7 Staender S, Kaufmann M, Scheidegger D. Human recoveries in the management of critical incidents in anesthesiology. Proc Am Nat Pat Assoc 1999.

8 Reason J. Managing the risks of organizational accidents. Hampshire: Ashgate Publishing Company; 1997.

9 Staender S, Kaufmann M, Scheidegger D. Critical Incident Reporting Systems in Anaesthesia. In: Vincent C, de Mol B (eds.). Safety in Medicine. Oxford: Pergamon, Elsevier Science; 2000. p. $65-82$.

10 Brunner HH, Conen D, Günter P, von Gunten M, Huber F, Kehrer B, et al. A safe healthcare system. Proposal for a national programme on patient safety improvement for Switzerland: www.swiss-q.org/apr-2001/docs/ Final_ReportE.pdf.

11 Barach P, Small SD. Reporting and preventing medical mishaps: lessons from non-medical near miss reporting systems. Br Med J 2000;320: 759-63.

12 Kohn LT, Corrigan JM, Donaldson MS (eds.). To err is human. Building a safer health system. Washington: National Academy Press; 1999.

13 Nolan TW. System changes to improve patient safety. Br Med J 2000;320:771-3. 